\title{
On the tree-width of even-hole-free graphs
}

\author{
Pierre Aboulker ${ }^{*} \quad$ Isolde Adler ${ }^{\dagger} \quad$ Eun Jung Kim ${ }^{\ddagger}$ \\ Ni Luh Dewi Sintiari $\S \quad$ Nicolas Trotignon $\S$
}

\begin{abstract}
The class of all even-hole-free graphs has unbounded tree-width, as it contains all complete graphs. Recently, a class of (even-hole, $K_{4}$ )-free graphs was constructed, that still has unbounded tree-width [Sintiari and Trotignon, 2019]. The class has unbounded degree and contains arbitrarily large clique-minors. We ask whether this is necessary.

We prove that for every graph $G$, if $G$ excludes a fixed graph $H$ as a minor, then $G$ either has small tree-width, or $G$ contains a large wall or the line graph of a large wall as induced subgraph. This can be seen as a strengthening of Robertson and Seymour's excluded grid theorem for the case of minor-free graphs. Our theorem implies that every class of even-hole-free graphs excluding a fixed graph as a minor has bounded tree-width. In fact, our theorem applies to a more general class: (theta, prism)-free graphs. This implies the known result that planar even hole-free graph have bounded tree-width [da Silva and Linhares Sales, Discrete Applied Mathematics 2010].

We conjecture that even-hole-free graphs of bounded degree have bounded tree-width. If true, this would mean that even-hole-freeness is testable in the bounded-degree graph model of property testing. We prove the conjecture for subcubic graphs and we give a bound on the
\end{abstract}

*DIENS, École normale supérieure, CNRS, PSL University, Paris, France. Email: pierreaboulker@gmail.com.

Supported by grant ANR-19-CE48-0016 from the French National Research Agency (ANR).

${ }^{\dagger}$ School of Computing, University of Leeds, Leeds, LS2 9JT, UK. Email: i.m.adler@leeds.ac.uk.

This research was partly carried out during a visit to Université Paris Dauphine, funded by LAMSADE.

${ }^{\ddagger}$ Université Paris-Dauphine, PSL University, CNRS UMR7243, LAMSADE, Paris, France. Email: eun-jung.kim@dauphine.fr.

Supported by the grant from the French National Agency under JCJC program (ASSK: ANR-18-CE40-0025-01).

${ }^{\S}$ Univ Lyon, EnsL, UCBL, CNRS, LIP, F-69342, LYON Cedex 07, France. Email: \{nicolas.trotignon,ni-luh-dewi.sintiari\}@ens-lyon.fr.

Partially supported by the LABEX MILYON (ANR-10-LABX-0070) of Université de Lyon, within the program 'Investissements d'Avenir' (ANR-11-IDEX-0007) operated by the French National Research Agency (ANR). 
tree-width of the class of (even hole, pyramid)-free graphs of degree at most 4 .

Keywords Even-hole-free graphs, grid theorem, tree-width, boundeddegree graphs, property testing

2012 ACM Subject Classification Theory of computation $\rightarrow$ Mathematics of computing $\rightarrow$ Discrete mathematics $\rightarrow$ Graph Theory; Theory of computation $\rightarrow$ Design and analysis of algorithms $\rightarrow$ Streaming, sublinear and near linear time algorithms

\section{Introduction}

Here, all graphs are simple and undirected. A hole in a graph is an induced cycle of length at least 4 . It is even or odd according to the parity of its length, that is the number of its edges. We say that a graph $G$ contains a graph $H$ if some induced subgraph of $G$ is isomorphic to $H$. A graph is $H$-free if it does not contain $H$. When $\mathcal{H}$ is a set of graphs, $G$ is $\mathcal{H}$-free if $G$ contains no graph of $\mathcal{H}$. A graph is therefore even-hole-free if it does not contain an even hole.

Even-hole-free graphs were the object of much attention, see for instance the survey [24]. However, many questions about them remain unanswered, such as the existence of a polynomial time algorithm to color them, or to find a maximum stable set. In fact, to the best of our knowledge, no problem that is polynomial time solvable for chordal graphs is known to be NPhard for even-hole-free graphs (where a chordal graph is a hole-free graph). Despite the existence of several decomposition theorems or structural properties (see [24]), no structure theorem is known for even-hole-free graphs.

In addition, motivated by the question whether even-hole-freeness is testable in the bounded degree model of property testing, the structure of even-hole-free graphs of bounded maximum degree is of interest. If evenhole-free graphs of bounded degree have bounded tree-width, it would imply testability in the bounded degree model, because even-hole-freeness is expressible in monadic second-order logic with modulo counting (CMSO) and CMSO is testable on bounded tree-width [1]. We will discuss this in greater detail below. Let us first provide some more background.

Background. We begin by recalling known definitions and results about tree-width. The clique number of a graph $G$, denoted by $\omega(G)$, is the maximum number of pairwise adjacent vertices in $G$. The tree-width of a graph $G$ is the minimum of $\omega(J)-1$ over all chordal graphs $J$ such that $G$ is a subgraph of $J$. The tree-width can be seen as a measure of the structural tameness of a graph: the smaller the tree-width, the more 'tree-like' the graph. A celebrated result [9] asserts that many problems (including graph 
coloring or finding a maximum stable set) can be solved in polynomial time when restricted to graphs of bounded tree-width. However, many graphs with in some sense a simple structure have large tree-width. For instance the complete graph on $n$ vertices, that we denote by $K_{n}$, has tree-width $n-1$. A graph $H$ is a minor of a graph $G$, if $H$ can be obtained from a subgraph of $G$ by contracting edges. Tree-width is monotone under taking minors in the sense that if $H$ is a minor of $G$, then the tree-width of $H$ is less than or equal to the tree-width of $G$. It follows that graphs that contain $K_{n}$ as a minor have tree-width at least $n-1$. The converse is not true: grids have arbitrarily large tree-width but they do not contain $K_{5}$ as a minor.

The class of all even-hole-free graphs trivially has unbounded tree-width, as is contains all complete graphs. Also, chordal graphs form a well studied subclass of even-hole-free graphs of unbounded tree-width. However, evenhole-free graphs with no triangle have bounded tree-width [7]. This leads to asking whether even-hole-free graphs of bounded clique number have bounded tree-width - a question that is first asked and motivated in [6]. This was answered negatively in [22], where (even hole, $K_{4}$ )-free graphs of arbitrarily large tree-width are described. However, the construction uses vertices of large degree and a large clique minor to increase the tree-width, and it seems natural to ask whether this is necessary.

It is known that planar even-hole-free graphs have bounded tree-width [21], and planar graphs do not contain $K_{\ell}$ as a minor for $\ell \geq 5$. Besides that, it is known that an upper bound on the length of the largest induced cycle implies an upper bound on the tree-width for graphs of bounded maximum degree [4].

Our contributions. The results explained above suggest the following two conjectures.

Conjecture 1. There is a function $f: \mathbb{N} \rightarrow \mathbb{N}$ such that every even-hole-free graph not containing $K_{\ell}$ as a minor has tree-width at most $f(\ell)$.

Conjecture 2. There is a function $f: \mathbb{N} \rightarrow \mathbb{N}$ such that every even-hole-free graph of degree at most $d$ has tree-width at most $f(d)$.

In this paper we prove Conjecture 1 (cf. Section 3). Indeed, we prove the following stronger result, which implies Conjecture 1 .

Theorem 1.1 (Induced grid theorem for minor-free graphs). For every graph $H$ there is a function $f_{H}: \mathbb{N} \rightarrow \mathbb{N}$ such that every $H$-minor-free graph of tree-width at least $f_{H}(k)$ contains $a(k \times k)$-wall or the line graph of a chordless $(k \times k)$-wall as an induced subgraph.

Here a wall is a (possibly subdivided) hexagonal grid (cf. Section 3).

Slightly more generally, Theorem 1.1 implies that (theta, prism)-free graphs (to be defined in Section 2) exluding a fixed minor have bounded 
tree-width. Note that (theta, prism)-free graphs form a superclass of evenhole-free graphs.

Our theorem can be seen as 'induced' version on minor-free graphs classes of the following famous theorem.

Theorem 1.2 (Robertson and Seymour [20]). There is a function $f: \mathbb{N} \rightarrow \mathbb{N}$ such that every graph of tree-width at least $f(k)$ contains $a(k \times k)$-wall as a subgraph.

Theorem 1.2 cannot be strengthened to finding walls as induced subgraphs in general, because the complete graph $K_{n}$ has tree-width $n-1$ and only contains complete graphs as induced subgraphs.

Note that graphs with no induced subdivision of a $6 \times 3$ wall, arbitrarily large tree-width and girth (in particular triangle and square-free) exist, as shown in 22]. But as for the construction of (even hole, $K_{4}$ )-free graphs of unbounded tree-width in [22], vertices of large degree and large clique minors are needed, we make the following conjecture.

Conjecture 3. For every $d \in \mathbb{N}$ there is a function $f_{d}: \mathbb{N} \rightarrow \mathbb{N}$ such that every graph with degree at most $d$ and tree-width at least $f_{d}(k)$ contains a $(k \times k)$-wall or the line graph of a $(k \times k)$-wall as an induced subgraph.

Conjecture 3 implies Conjecture 2, Conjecture 3 is wide open, and our results can be seen as a step in the direction of a proof.

For Conjecture 2 (that is trivial for $l \leq 2$ ), we give a proof for $l=3$ by providing a full structural description of subcubic even-hole-free graphs (a graph is subcubic if it does not contain a vertex of degree more than 3 ). In fact, all these results apply to (theta, prism)-free graphs (cf. Section 2 for the details). We also prove a weakening of Conjecture 2 for $l=4$ (cf. Section 5 ).

Motivation from property testing. Our other source of motivation for studying even-hole-free graphs of bounded degree stems from the question whether even-hole-freeness is testable in the bounded degree graph model. Motivated by the growing need of highly efficient algorithms, in particular when the inputs are huge, property testing aims at devising sublinear time algorithms. Property testing algorithms (simply called testers) solve a relaxed version of decision problems, they are randomised, and they come with a small controllable error probability.

Since the input cannot be read even once in sublinear time, testers have local access to the input graph only. The bounded degree graph model assumes a fixed upper bound $d$ on the degree of all graphs, and the testers proceed by sampling a constant number of vertices of the input graph and exploring their local (constant radius) neighborhoods. A property $P$ is testable, if there is an $\epsilon$-tester for $P$, for every fixed small $\epsilon>0$. For an input graph 
$G$, an $\epsilon$-tester determines, with probability at least $2 / 3$ correctly, whether $G$ has property $P$, or $G$ is $\epsilon$-far from having property $P$. Here a property is simply an isomorphism closed class of graphs. A graph $G$ is $\epsilon$-close to $P$, if there is a graph $G^{\prime} \in P$ on the same number $n$ of vertices as $G$, such that $G$ and $G^{\prime}$ can be made isomorphic by at most $\epsilon d n$ edge modifications (deletions or insertions) in $G$ or $G^{\prime}$, and otherwise, $G$ is $\epsilon$-far from $P$. The number of vertices explored in the input graph is called the query complexity of the tester. The model requires testers to have constant query complexity.

Properties that are known to be testable in the bounded degree graph model include subgraph-freeness (for a fixed subgraph), $k$-edge connectivity, cycle-freeness, being Eulerian, degree-regularity [14, bounded tree-width and minor-freeness [3, 15, 18, hyperfinite properties [19], $k$-vertex connectivity [25, 12], and subdivision-freeness [17]. Properties that are not testable in the bounded degree model include bipartiteness, 3-colorablilty, expansion properties, and $k$-clusterability, cf. [13].

Since the testers can only explore a constant number of constant radius neighborhoods in the input graph, intuitively, properties that are testable should have some form of 'local' nature. Hence it might seem unlikely that properties like Hamiltonicity and even-hole-freeness are testable, due to the large cycles involved. Indeed, it can be shown that there is no one-sided error tester for Hamiltonicity (i. e. there is no tester that always accepts yesinstances) with constant query complexity. More precisely, every one-sided tester has query complexity at least $\Omega(n)$ [16].

Perhaps surprisingly, our results suggest a different picture for evenhole-freeness. It is known that on graphs of bounded degree and bounded tree-width, every property that can be expressed in monadic second-order logic with counting (CMSO), is testable with constant query complexity and polylogarithmic running time [1]. (Here polylogarithmic in $n$ means bounded by a polynomial in $\log n$.) It is straightforward to see that evenhole-freeness is expressible in CMSO. This can be done by expressing that there is no set $X$ of edges that form an induced hole, where $|X|$ is even (cf. eg. [10] for more details on CMSO expressibility). Together with the fact that bounded tree-width is testable, this implies that if Conjecture 2 is true, then even-hole-freeness is testable with constant query complexity and polylogarithmic running time. (This is done by first testing for bounded tree-width and, if the answer is positive, testing for even-hole-freeness using CMSO testability.) Our results in Section 4 imply the following.

Theorem 1.3. On subcubic graphs, even-hole-freeness is testable with constant query complexity and polylogarithmic running time.

Structure of the paper. We start with fixing notation in Section2, Section 3 contains the proof of the induced grid theorem for minor-free graph 
classes, and the proof of Conjecture 1. Section 4 contains the structure theorem for subcubic (theta, prism)-free graphs and the proof of Conjecture 2 for $d=3$. Recall that (theta, prism)-free graphs are defined in the next section, and it is a superclass of even-hole-free graphs. In Section 5 we provide a structure theorem for (even hole, pyramid)-free graphs of maximum degree 4 , and we derive a bound on the tree-width of this class (pyramids will be defined in the next section). In Section 6 we give ideas suggesting that a structure theorem for even-hole-free graphs with maximum degree 4 might exist, and if so, should imply bounded tree-width.

\section{Notation}

We let $\mathbb{N}$ denote the set of natural numbers including 0 . We use $X \sqcup Y$ instead of $X \cup Y$ if $X \cap Y=\emptyset$. We use $(0,1]$ to denote the real interval that excludes 0 and includes 1 . For any $n \in \mathbb{N}, n \geq 1$, let $[n]:=\{1, \ldots, n\}$.

An (undirected) graph is a pair $G=(V(G), E(G))$, consisting of a set $V(G)$, the set of vertices of $G$, and a set $E(G)$ of edges of $G$, where an edge is a two-vertex subset of $V(G)$. A graph $H$ is a subgraph of a graph $G$, if $V(H) \subseteq V(G)$ and $E(H) \subseteq E(G)$. For a set $X \subseteq V(G)$ the subgraph induced by $X$ in $G$ is the subgraph $G[X]$ of $G$ with vertex set $X$, such that $e \in E(G[X])$ iff $e \in E(G)$ and $e \subseteq X$. A graph $H$ is an induced subgraph of $G$, if $H=G[X]$ for some $X \subseteq V(G)$. For a set $S \subseteq V(G)$ we let $G \backslash S:=G[V(G) \backslash S]$ and if $S=\{v\}$ is a singleton set, then we write $G \backslash v$ instead of $G \backslash\{v\}$.

A path in $G$ is a sequence $P$ of distinct vertices $p_{1} \ldots p_{n}$, where for $i, j \in\{1, \ldots, n\}, p_{i} p_{j} \in E(G)$ if and only if $|i-j|=1$. For two vertices $p_{i}, p_{j} \in V(P)$ with $j>i$, the path $p_{i} p_{i+1} \ldots p_{j}$ is a subpath of $P$ that is denoted by $p_{i} P p_{j}$. The subpath $p_{2} \ldots p_{n-1}$ is called the interior of $P$. The vertices $p_{1}, p_{n}$ are the ends of the path, and the vertices in the interior of $P$ are called the internal vertices of $P$. A cycle is defined similarly, with the additional properties that $n \geq 4$ and $p_{1}=p_{n}$. The length of a path $P$ is the number of edges of $P$. The length of cycle is defined similarly. Let $G$ be a graph. For vertices $u, v \in V(G)$, the distance between $u$ and $v$ in $G$, denoted by $\operatorname{dist}_{G}(u, v)$, is the length of a shortest path from $u$ to $v$, if a path exists, and $\infty$ otherwise. For two subsets $X, Y \subseteq V(G)$, the distance between $X$ and $Y$ is $\min \left\{\operatorname{dist}_{G}(x, y) \mid x \in X, y \in Y\right\}$. For $v \in V(G)$, we call the set $N_{r}^{G}(v):=\left\{w \in V(G) \mid \operatorname{dist}_{G}(v, w) \leq r\right\}$ the $r$-neighborhood of $v$ (in $G)$.

The degree of a vertex $v$ in $G$ is defined as $\operatorname{deg}_{G}(v):=\mid\{u \in V(G) \mid$ $\{u, v\} \in E(G)\} \mid$. The degree of $G, \operatorname{deg}(G)$, is the maximum degree over all vertices of $G$. By $\mathcal{C}_{d}$ we denote the class of all graphs of degree at most $d \in \mathbb{N}$.

The line graph of a graph $G$ is the graph $L(G)$, with $V(L(G))=E(G)$ 
and two vertices of $L(G)$ are adjacent, if their corresponding edges are incident in $G$. An edge $e \in E(G)$ is a chord of cycle $C$, if the endpoints of $e$ are vertices of $C$ that are not adjacent on $C$. A hole is a chordless cycle of length at least 4. A clique in $G$ is a set $X \subseteq V(G)$ of vertices such that $\{v, w\} \in E(G)$ for every pair $v, w \in X$ with $v \neq w$. A graph $K$ is complete, if $V(K)$ is a clique in $K$. We use $K_{k}$ to denote the complete graph on $k$ vertices. For disjoint sets $A, B \subseteq V(G)$, we say that $A$ is anticomplete to $B$ if no edges are present between $A$ and $B$ in $G$.

A pyramid is a graph made of three chordless paths $P_{1}=x \ldots a, P_{2}=$ $x \ldots b, P_{3}=x \ldots c$, each of length at least 1 , two of which have length at least 2 , internally vertex-disjoint, and such that $a b c$ is a triangle and no edges exist between the paths except those of the triangle and the three edges incident to $x$. The vertex $x$ is called the apex of the pyramid.

A prism is a graph made of three vertex-disjoint chordless paths $P_{1}=$ $a \ldots a^{\prime}, P_{2}=b \ldots b^{\prime}, P_{3}=c \ldots c^{\prime}$ of length at least 1 , such that $a b c$ and $a^{\prime} b^{\prime} c^{\prime}$ are triangles and no edges exist between the paths except those of the two triangles.

A thet $a$ is a graph made of three internally vertex-disjoint chordless paths $P_{1}=a \ldots b, P_{2}=a \ldots b, P_{3}=a \ldots b$ of length at least 2 and such that no edges exist between the paths except the three edges incident to $a$ and the three edges incident to $b$.

A wheel is a graph formed from a hole $H$ together with a vertex $x$ that has at least three neighbors in the hole. Such a hole $H$ is called the rim, and such a vertex $x$ is called the center of the wheel. We denote by $(H, x)$, the wheel with rim $H$ and center $x$.
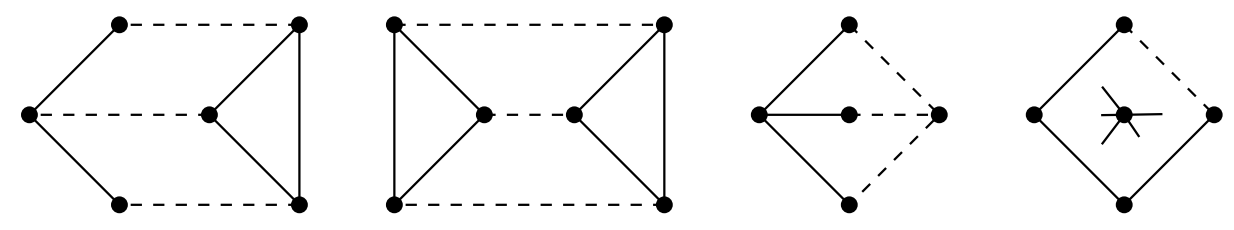

Figure 1: Pyramid, prism, theta, and wheel (dashed lines represent paths)

Theta and prism are relevant in this work, because of the following wellknown lemma. The following lemma clearly implies that (theta, prism)-free graphs form a superclass of even-hole-free graphs.

Lemma 2.1. Every theta and every prism contains an even hole.

PROOF - It follows by the fact that there exist two paths in a theta or in a prism that have same parity, which induce an even hole. 


\section{Even-hole-free graphs excluding a minor}

In this section we prove an 'induced grid theorem' for graphs excluding a fixed minor. From this we derive that even-hole-free graphs excluding a fixed minor have bounded tree-width.

We begin by defining grids and walls. Let $n, m$ be integers with $n, m \geq 2$. An $(n \times m)$-grid is the graph $G_{n \times m}$ with $V\left(G_{n \times m}\right)=[n] \times[m]$ and $E\left(G_{n \times m}\right)=\left\{\left\{\left(i_{1}, j_{1}\right),\left(i_{2}, j_{2}\right)\right\}|| i_{1}-i_{2}|+| j_{1}-j_{2} \mid=1, i_{1}, i_{2} \in[n], j_{1}, j_{2} \in[m]\right\}$.

Figure 2 shows $G_{5 \times 5}$.

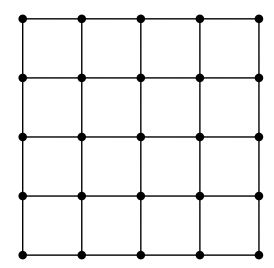

Figure 2: The $(5 \times 5)$-grid $G_{5 \times 5}$.

Let $n, m \geq 2$ be integers. An elementary $(n \times m)$-wall is a graph $G=$ $(V, E)$ with vertex set

$$
\begin{aligned}
V= & \{(1,2 j-1) \mid 1 \leq j \leq m\} \cup\{(i, j) \mid 1<i<n, 1 \leq j \leq 2 m\} \\
& \cup\{(n, 2 j-1) \mid 1 \leq j \leq m, \text { if } n \text { is even }\} \cup\{(n, 2 j) \mid 1 \leq j \leq m, \text { if } n \text { is odd }\}
\end{aligned}
$$

and edge set

$$
\begin{aligned}
E= & \{(1,2 j-1),(1,2 j+1) \mid 1 \leq j \leq m-1\} \cup\{\{(i, j),(i, j+1)\} \mid 2 \leq i<n, 1 \leq j<2 m\} \\
& \cup\{\{(n, 2 j),(n, 2 j+2))\} \mid 1 \leq j<m \text { if } n \text { is odd }\} \\
& \cup\{\{(n, 2 j-1),(n, 2 j+1)\} \mid 1 \leq j<m \text { if } n \text { is even }\} \\
& \cup\{\{(i, j),(i+1, j)\} \mid 1 \leq i<n, 1 \leq j \leq 2 m, i, j \text { odd }\} \\
& \cup\{\{(i, j),(i+1, j)\} \mid 1 \leq i<n, 1 \leq j \leq 2 m, i, j \text { even }\} .
\end{aligned}
$$

Figure 3 shows an elementary $(5 \times 5)$-wall.

An elementary $(n \times m)$-wall has $n$ horizontal paths, where the first horizontal path is induced by the vertex set $\{(1,2 j-1) \mid 1 \leq j \leq 2 m\}$, the $i$ th horizontal path is induced by the vertex set $\{(i, j) \mid 1 \leq j \leq 2 m\}$. for $1<i<n$, and the $n$th horizontal path is induced by the vertex set $\{(n, 2 j-1) \mid 1 \leq j \leq 2 m\}$ if $n$ is odd, and by $\{(n, 2 j) \mid 1 \leq j \leq 2 m\}$ if $n$ is even. An elementary $(n \times m)$-wall has $m$ vertical paths, where the $j$ th vertical path is induced by the vertex set $\{(i, 2 j-1),(i+1,2 j-1) \mid 1 \leq i<$ $n, i$ odd $\} \cup\{(i, 2 j),(i+1,2 j) \mid 1 \leq i<n, i$ even $\}$, for $1 \leq j \leq m$. Figure 4 shows an elementary wall with vertical paths. 


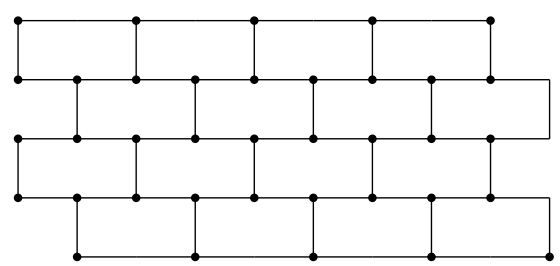

Figure 3: The elementary $(5 \times 5)$-wall.

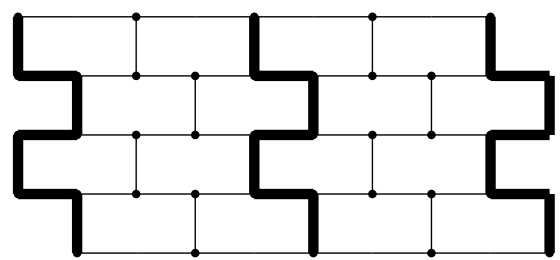

Figure 4: An elementary $(5 \times 5)$-wall with three of the five vertical paths highlighted.

An $(n \times m)$-wall is a subdivision of an elementary $(n \times m)$-wall. An $(n \times m)$-wall also has $n$ horizontal paths and $m$ vertical paths, which arise from the paths of the underlying elementary wall including the subdivision vertices.

Robertson and Seymour's famous excluded grid theorem states that for any graph $G$, either $G$ has small tree-width or $G$ contains a large grid minor, thus identifying large grid minors as canonical obstructions to small treewidth.

Theorem 3.1 (Robertson and Seymour [20]). There is a function $f$ such that for every $k \geq 1$ and every graph $G$, if $\operatorname{tw}(G) \geq f(k)$ then $G$ contains the $(k \times k)$-grid as a minor.

The theorem continues to hold if we replace grid by wall, and the latter have the advantage of having a maximum degree of three, which allows finding walls as subgraphs. Recall Theorem 1.2 .

Theorem 1.2 (Robertson and Seymour [20]). There is a function $f: \mathbb{N} \rightarrow \mathbb{N}$ such that every graph of tree-width at least $f(k)$ contains $a(k \times k)$-wall as a subgraph.

Obviously, this cannot be strengthened to finding walls as induced subgraphs, because the complete graph $K_{n}$ has tree-width $n-1$ and only contains complete graphs as induced subgraphs. A graph $G$ is called chordless, if no cycle of length at least 4 in $G$ has a chord in $G$. For graphs excluding a fixed minor, we now prove an 'induced grid theorem'. Recall Theorem 1.1 .

Theorem 1.1 (Induced grid theorem for minor-free graphs). For every graph $H$ there is a function $f_{H}: \mathbb{N} \rightarrow \mathbb{N}$ such that every $H$-minor-free graph 
of tree-width at least $f_{H}(k)$ contains $a(k \times k)$-wall or the line graph of a chordless $(k \times k)$-wall as an induced subgraph.

Observe that the line-graph of a chordless wall has degree at most 3 . For the proof of Theorem 1.1 we need some lemmas and notation. A fork is a tree with exactly three leaves. A semi-fork is a graph obtained from a triangle by appending disjoint paths of length at least 1 at each vertex of the triangle. Note that both a fork and a semi-fork have precisely three degree-one vertices.

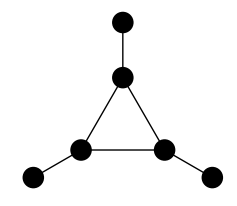

Figure 5: The net graph.

A semi-fork obtained from a triangle by appending disjoint paths of length exactly 1 at each vertex of the triangle is called a net graph (cf. Figure 5). Let $G$ be a graph and let $v \in V(G)$ be a vertex of degree 3 in $G$ with neighbors $a, b, c$. The graph obtained by a net graph replacement at $v$ is the graph $H$, with $V(H)=(V(G) \backslash\{v\}) \cup\{x, y, z\}$, where $x, y, z$ are new vertices, i. e. $x, y, z \notin V(G)$, and $E(H)=E(G \backslash v) \cup\{x y, y z, x z\} \cup\{x a, y b, z c\}$.

The 'grid-like' configurations we aim for are walls and line graphs of walls. On the way, we also encounter a slightly untidier 'mix' of both, that we call stone wall. An $(n \times m)$-stone wall a graph obtained from a wall $W$ by picking a (possibly empty) subset $X$ of the degree-3-vertices of $W$ and performing net graph replacements at each vertex in $X$. Note that if we perform net graph replacements at all degree-3-vertices of the wall $W$, the resulting graph is the line graph of a wall (namely of the wall $W^{\prime}$ obtained from $W$ by adding an additional subdivision vertex on each path that connects two degree-3-vertices). Observe that stone walls have maximum degree 3. A stone wall is homogeneous, if it is either a wall or the line graph of a wall. Note that because stone walls have degree at most 3 , if a homogeneous stone wall is the line graph of a wall $W, W$ must be chordless.

A triangulated $(n \times m)$-grid is the graph $G_{n \times m}^{\triangle}$ with $V\left(G_{n \times m}^{\triangle}\right)=V\left(G_{n \times m}\right)$ and $E\left(G_{n \times m}^{\triangle}\right)=E\left(G_{n \times m}\right) \cup\{\{(i, j),(i-1, j+1)\} \mid 1<i \leq m, 1 \leq j<m\}$, see Figure 6. The vertices $(1,1),(1, m),(n, 1)$ and $(n, m)$ are called the corners of the (triangulated) grid.

The following lemma shows that a large wall contains many smaller induced subwalls.

Lemma 3.2. For $n, m \in \mathbb{N}$, let $W$ be an $(n \times m)$-wall. Let $X$ be a set of pairwise non-adjacent rows of $W$ and let $Y$ be a set of pairwise non- 


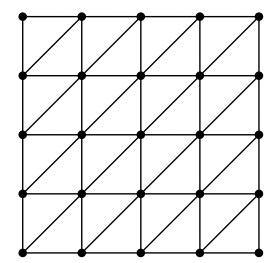

Figure 6: The triangulated $(5 \times 5)$-grid $G_{5 \times 5}^{\triangle}$.

adjacent columns of $W$. Then $W$ contains an induced $(|X| \times|Y|)$-wall $W^{\prime}$, with $V\left(W^{\prime}\right) \subseteq \bigcup_{P \in X} V(P) \cup \bigcup_{Q \in X} V(Q)$.

Proof - Assume that $|X| \geq 2$ and $|Y| \geq 2$. We obtain $W^{\prime}$ by taking the subgraph of $W$ induced by the set $\bigcup_{P \in X} V(P) \cup \bigcup_{Q \in X} V(Q)$ and repeatedly deleting degree-1-vertices until all vertices have degree at least 2.

For the proof of Theorem 1.1, we use a corollary of the main result of [11], and we need the notion of contraction. Let $G$ and $H$ be graphs. If $H$ can be obtained from $G$ by a sequence of edge contractions, then $H$ is called a contraction of $G$. Alternatively, contractions can be defined via mappings as follows. Let $G$ and $H$ be graphs and let $\varphi: V(G) \rightarrow V(H)$ be a surjective mapping such that

1. for every vertex $v \in V(H)$, its pre-image $\varphi^{-1}(v)$ is connected in $G$,

2. for every edge $u v \in E(H)$, the graph $G\left[\varphi^{-1}(u) \cup \varphi^{-1}(v)\right]$ is connected,

3. for every edge $x y \in E(G)$, either $\varphi(u)=\varphi(v)$ or $\varphi(u) \varphi(v) \in E(H)$.

Corollary 3.3 (Fomin, Golovach and Thilikos [11]). Let $H$ be a graph and let $G$ be a graph excluding $H$ as a minor. There exists a constant $c_{H}$ such that if $\operatorname{tw}(G) \geq c_{H} \cdot(k+1)^{2}$, then $G$ contains an induced subgraph that contains $G_{k \times k}^{\triangle}$ as a contraction.

The following lemma will help us to find a large stone wall in a graph containing a large triangulated grid as a contraction.

Lemma 3.4. Let $G$ be a connected graph whose vertex set is partitioned into connected sets $A, A^{\prime}, B, B^{\prime}, C, C^{\prime}$ and $S$. Suppose that every edge of $G$ has either both ends in one of the sets, or is from $A^{\prime}$ to $A$, from $B^{\prime}$ to $B$, from $C^{\prime}$ to $C$, or from $S$ to $A \cup B \cup C$.

If $a \in A^{\prime}, b \in B^{\prime}$ and $c \in C^{\prime}$, then $a, b$ and $c$ are the degree one vertices of some induced fork or semi-fork of $G$.

ProOF - Let $P$ be a shortest path from $b$ to $c$ in $G\left[B^{\prime} \cup B \cup S \cup C \cup C^{\prime}\right]$. Note that $P$ must go through $S$. Let $Q=a \ldots w$ in $G\left[A^{\prime} \cup A \cup S\right]$ be a shortest path such that $w$ has some neighbors in $P$. Let $u$ (resp. $v$ ) be the 
neighbor of $w$ in $P$ closest to $b$ (resp. to $c$ ) along $P$. Note that $u \neq b$ and $v \neq c$. If $u=v$, then $P$ and $Q$ form a fork. If $u$ is adjacent to $v$, then $P$ and $Q$ form a semi-fork. If $u \neq v$ and $u v \notin E(G)$, then $a P w, b Q u$ and $c Q v$ form a fork. In all cases, $a, b$ and $c$ are the three vertices of degree 1 of the fork or semi-fork.

For tidying up stone walls, we make use of a natural variant of Ramsey's Theorem for bipartite graphs, first introduced by Beineke and Schwenk in 1975.

Theorem 3.5 (Beineke and Schwenk [2]). For every integer $r \geq 1$ there exists a smallest positive integer $n=n(r)$, such that any 2-edge-coloring of the complete bipartite graph $K_{n, n}$ contains a monochromatic $K_{r, r}$.

In [23] it was shown that $n(r) \leq 2^{r}(r-1)+1$.

The next lemma shows that any sufficiently large stone wall also contains a large homogeneous stone wall as induced subgraph.

Lemma 3.6. For every integer $r \geq 2$ there exists an integer $n=n(r)$ such that every $(n \times n)$-stone wall contains a homogeneous $(r \times r)$-stone wall as induced subgraph.

PROOF -

Given $r$, let $n=n(r)$ be large enough. Given an $(n \times n)$-stone wall $W$, we define an auxiliary wall $W^{\prime}$, which is obtained from $W$ by contracting every triangle. Each vertex in $W^{\prime}$ that is the result of contracting a triangle is colored red (red encodes 'semi-fork'), and all other degree-3-vertices of $W^{\prime}$ are colored green (green encodes 'fork').

Define a complete bipartite graph $H$ with $V(H)=A \cup B$ as follows. The elements of $A$ are horizontal paths of $W^{\prime}$, and the elements of $B$ are the vertical paths in $W^{\prime}$. Note that each vertical path has two colored vertices in common with each horizontal path.

We fix an orientation of the horizontal paths 'from left to right'. Now we color the edges of $H$ with four colors. Let $P \in A$ be a horizontal path and let $Q \in B$ be a vertical path.

1. If $V(P) \cap V(Q) \subseteq V\left(W^{\prime}\right)$ contains two green vertices, we color the edge $P Q$ green.

2. If $V(P) \cap V(Q) \subseteq V\left(W^{\prime}\right)$ contains two red vertices, we color the edge $P Q$ red.

3. If $V(P) \cap V(Q) \subseteq V\left(W^{\prime}\right)$ contains a green and a red vertex, and the green vertex appears before the red vertex when traversing $P$ from left to right, then we color the edge $P Q$ white.

4. If $V(P) \cap V(Q) \subseteq V\left(W^{\prime}\right)$ contains a green and a red vertex, and the red vertex appears before the green vertex when traversing $P$ from left to right, then we color the edge $P Q$ black. 
By applying Theorem 3.5 (multiple times, if necessary), we find that $H$ contains a large monochromatic complete bipartite subgraph $H^{\prime}$.

If $H^{\prime}$ is green (or red, respectively), we find a large subwall in $W^{\prime}$ where all vertices of degree 3 are green (red, respectively) as follows. We take the horizontal and vertical paths in $W^{\prime}$ that correspond to $V\left(H^{\prime}\right)$, leaving out every second path to make sure that the horizontal paths we keep are pairwise non-adjacent, and that the vertical paths we keep are pairwise nonadjacent. Then we apply Lemma 3.2 . Undoing the contractions of triangles in the case that $H^{\prime}$ is red, we thus obtain a large induced homogeneous stone wall in $W$.

In the case that $H^{\prime}$ is white or black, we find a large subwall $W^{\prime \prime}$ in $W^{\prime}$ where both red and green appear at each intersection of a horizontal and a vertical path. W.l.o.g. assume that $H^{\prime}$ is white (otherwise flip the wall exchanging left and right). We will now explain how to find a large induced subwall of $W$.

Let $X$ be a maximal subset of horizontal paths of $W^{\prime \prime}$ of pairwise distance 10 , and let $Y$ be a maximal subset of vertical paths of $W^{\prime \prime}$ of pairwise distance 10. Whenever a path $P \in X$ and a path $Q \in Y$ intersect, we reroute the two paths locally around their intersection to avoid red vertices of degree 3 as follows.

Let $u, v, w, x$ be consecutive degree-3-vertices on $Q$ with $u, v$ on $P$. Assume $u, v, w, x$ appear in this order when walking along $Q$ from top to bottom, and wl.o.g. assume $u$ is red (otherwise walk along $Q$ from bottom to top). Since $H^{\prime}$ is white, $w$ is green $x$ is red. Now we reroute $P$ and $Q$ locally, such that after rerouting, both degree-3-vertices at the intersection of $P$ and $Q$ are green. The rerouting is shown in Figure 7. Note that there is enough space around the intersection, because we only use paths in $X \cup Y$.
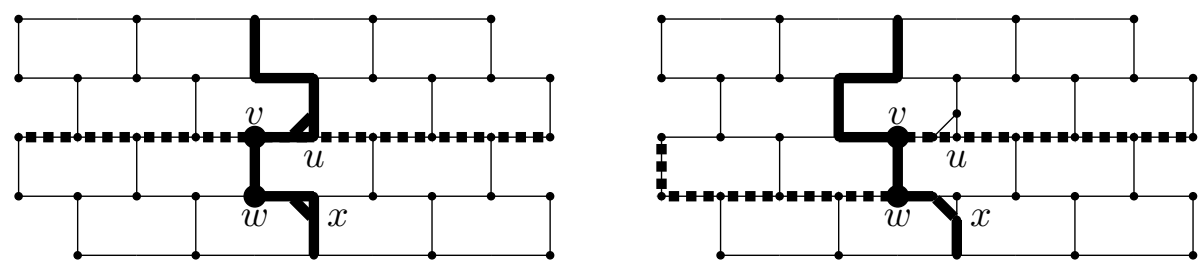

Figure 7: Rerouting in the proof of Lemma 3.6

Rerouting in this manner for every pair of paths in $X$ and $Y$, we end up with a large subwall of $W^{\prime}$ that is green, which is also an induced homogeneous stone wall in $W$.

PROOF - Proof of Theorem 1.1 Let $H$ be a graph and let $G$ be a graph excluding $H$ as a minor. We may assume $G$ is connected. Let $c_{H}$ be as in Corollary 3.3 and let $h \in \mathbb{N}$ be sufficiently large, and let $k=8 h$. Assume $\operatorname{tw}(G) \geq c_{H} \cdot(k+1)^{2}$. Then $G$ contains an induced subgraph $G^{\prime}$, such that $G^{\prime}$ 


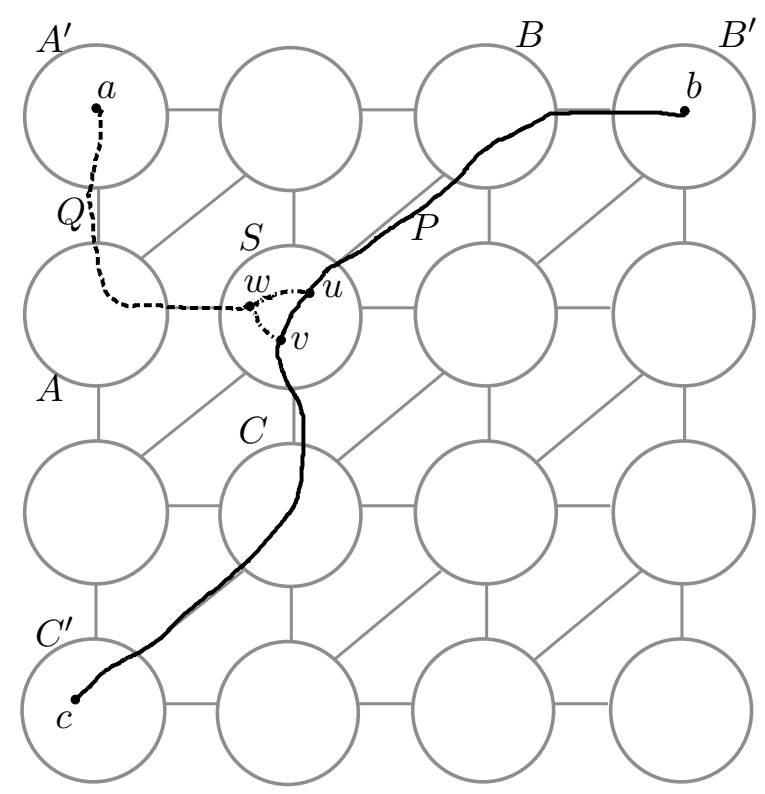

Figure 8: Proof of Theorem 1.1. Using Lemma 3.4 to find an induced fork or semi-fork in $G^{\prime}$.

contains $G_{k \times k}^{\triangle}$ as a contraction, witnessed by a contraction mapping $\varphi: G^{\prime} \rightarrow$ $G_{k \times k}^{\triangle}$. The graph $G_{k \times k}^{\triangle}$ contains $(2 h)^{2}$ graphs $G_{4 \times 4}^{\triangle}$. We pick every second row of graphs $G_{4 \times 4}^{\triangle}$, and every second graph $G_{4 \times 4}^{\triangle}$ of the row allows us to find an induced fork or an induced semi-fork in $G^{\prime}$ as follows. Assume the vertices of $G_{4 \times 4}^{\triangle}$ are $(1,1), \ldots,(4,4)$. Let $A^{\prime}:=\varphi^{-1}((1,1)), A:=\varphi^{-1}((2,1)), B^{\prime}:=$ $\varphi^{-1}((1,4)), B:=\varphi^{-1}((1,3)), C^{\prime}:=\varphi^{-1}((4,1)), C:=\varphi^{-1}((3,2))$, and $S:=$ $\varphi^{-1}((2,2))$. Lemma 3.4 yields a fork or a semi-fork in $G^{\prime}\left[A \cup A^{\prime} \cup B \cup B^{\prime} \cup\right.$ $\left.C \cup C^{\prime} \cup S\right]$ and hence in $G^{\prime}$ (cf. Figure 8).

These forks can be combined into a large stone wall by adding induced paths to connect the forks or semi-forks appropriately, cf. Figure 9 .

Hence $G$ contains a large stone wall as induced subgraph, and we can use Lemma 3.6 to complete the proof.

Let us remark that the function $f_{H}$ in Theorem 1.1 is computable.

Corollary 3.7. For every fixed graph $H$, the class of (theta, prism)-free graphs that do not contain $H$ as a minor has bounded tree-width. In particular, even-hole-free graphs that do not contain $H$ as a minor have bounded tree-width.

PROOF - A large wall contains a theta, and the line graph of a large wall contains a prism.

The following corollary reproves a theorem from [21]. 


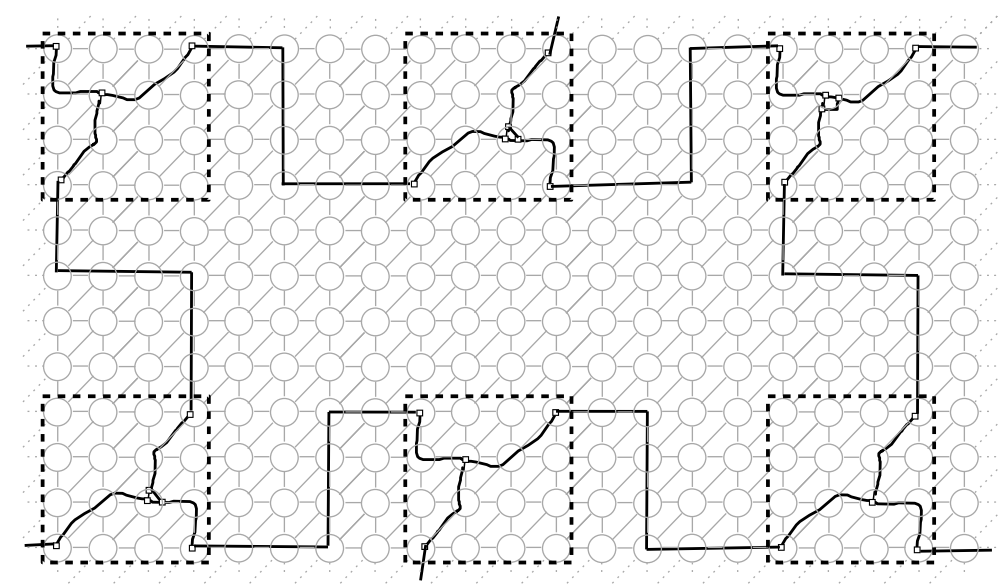

Figure 9: Proof of Theorem 1.1: Finding a stone wall in $G_{k \times k}^{\triangle}$.

Corollary 3.8. Planar even-hole-free graphs have bounded tree-width.

PROOF - This follows from Corollary 3.7 because planar graphs exclude $K_{5}$ as a minor.

\section{Subcubic (theta, prism)-free graphs}

In this Section, we prove that even-hole subcubic graphs can be described by a structure theorem, that implies tree-width at most 3 . In fact our result is for a more general class: (theta, prism)-free subcubic graphs.

A wheel that is not a pyramid is a proper wheel. A sector of a wheel $(H, x)$ is a subpath of $H$ whose endnodes are adjacent to $x$, and whose internal vertices are not.

An extended prism is a graph made of five vertex-disjoint chordless paths of length at least $1 A=a \ldots x, A^{\prime}=x \ldots a^{\prime}, B=b \ldots y, B^{\prime}=y \ldots b^{\prime}$, $C=c \ldots c^{\prime}$ such that $a b c$ is a triangle, $a^{\prime} b^{\prime} c^{\prime}$ is a triangle, $x y$ is an edge and no edges exist between the paths except $x y$ and those of the two triangles (see Figure 10).

A subset (possibly empty) of vertices $S \subseteq V(G)$ is a separator of $G$ if $G \backslash S$ contains at least two connected components. A clique separator is a separator $S$ that is a clique. 

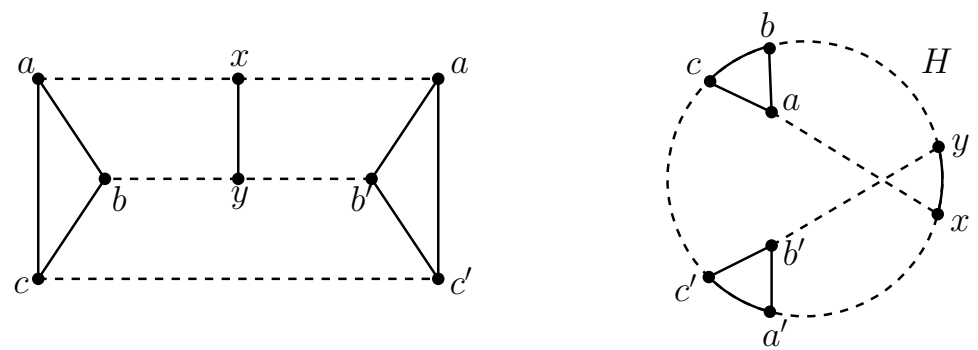

Figure 10: Two different drawings of an extended prism

A proper separation in a graph $G$ is a triple $(\{a, b\}, X, Y)$ satisfying the following.

(i) $\{a, b\}, X, Y$ are disjoint, non-empty and $V(G)=\{a, b\} \cup X \cup Y$.

(ii) There are no edges from $X$ to $Y$.

(iii) $a$ and $b$ are non-adjacent.

(iv) $a$ and $b$ have exactly two neighbors in $X$.

(v) $a$ and $b$ have exactly one neighbor in $Y$.

(vi) There exists a path from $a$ to $b$ with interior in $X$, and there exists a path from $a$ to $b$ with interior in $Y$.

(vii) $G[Y \cup\{a, b\}]$ is not a chordless path from $a$ to $b$.

A proper separator of $G$ is a pair $\{a, b\} \subseteq V(G)$ such that there exists a proper separation $(\{a, b\}, X, Y)$.

Let $\mathcal{C}$ be the class of (theta, prism)-free subcubic graphs. The cube is the graph made of a hole $v_{1} v_{2} \ldots v_{6} v_{1}$ and two non-adjacent vertices $x$ and $y$ such that $N_{H}(x)=\left\{v_{1}, v_{3}, v_{5}\right\}$ and $N_{H}(y)=\left\{v_{2}, v_{4}, v_{6}\right\}$. Call a graph in $\mathcal{C}$ basic if it is isomorphic to a chordless cycle, a clique of size at most 4 , the cube, a proper wheel, a pyramid, or an extended prism. An example of graph in $\mathcal{C}$ that is not basic is provided in Figure 11

We need the following lemma.

Lemma 4.1. Let $G$ be a theta-free subcubic graph, let $H$ be a hole in $G$, and $v \in G \backslash H$. Then $v$ has at most three neighbors in $H$, and if $v$ has exactly two neighbors in $H$, then they are adjacent.

Proof - Let $v \in G \backslash H$. Since $G$ is subcubic, $d_{H}(v) \leq 3$. If $v$ has exactly two neighbors in $H$, but they are non-adjacent then $G[H \cup\{v\}]$ would induce a theta, a contradiction.

The main theorem of this section is the following. 


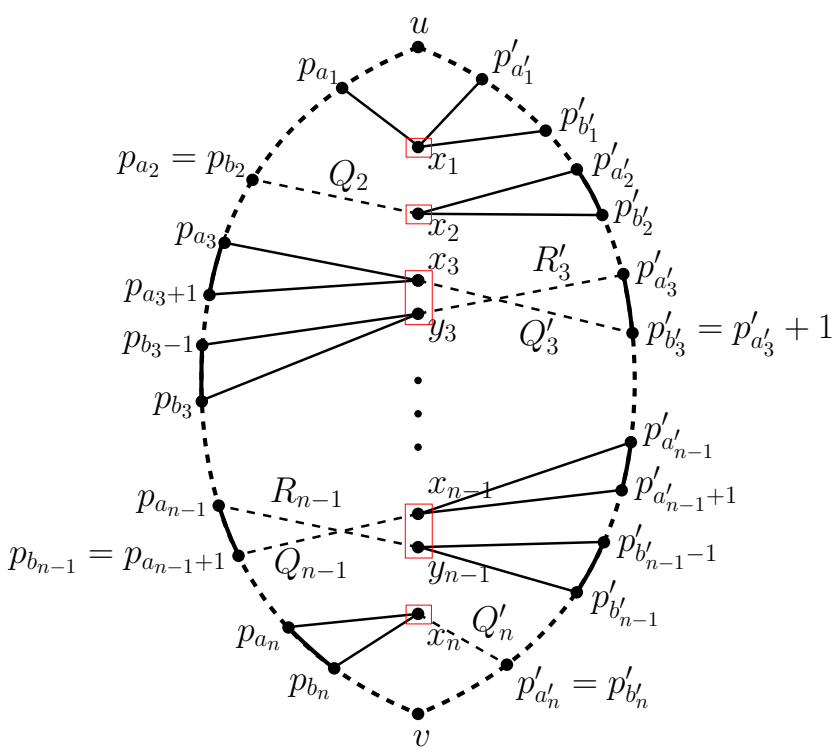

Figure 11: An example of non-basic graph in $\mathcal{C}$

Theorem 4.2. Let $G$ be a (theta, prism)-free subcubic graph. Then one of the following holds:

- $G$ is a basic graph;

- G has a clique separator of size at most 2;

- G has a proper separator.

PROOF — Let $G$ be a (theta, prism)-free subcubic graph. We may assume that $G$ has no clique separator (and is in particular connected for otherwise the empty set is a clique separator).

(1) We may assume that $G$ is ( $K_{4}$, cube)-free.

Proof of (1). If $G$ contains $K_{4}$, then since $G$ is a subcubic connected graph, $G=K_{4}$, so $G$ is basic. The proof is similar when $G$ contains the cube. This proves (1).

(2) We may assume that $G$ does not contain a proper wheel.

Proof of (2). Let $W=(H, x)$ be a proper wheel in $G$. Let $a, b, c$, be the three neighbors of $x$. We call $A$ ( $B, C$, resp.) the path of $H$ from $b$ to $c$ (from $a$ to $c$, from $a$ to $b$, resp.) that does not contain $a$ (b, $c$, resp.).

Suppose that some vertex $y$ of $G \backslash W$ has neighbors in the three sectors of $W$, say $a^{\prime}$ in $A, b^{\prime}$ in $B$, and $c^{\prime}$ in $C$. Hence, $a, c^{\prime}, b, a^{\prime}, c$, and $b^{\prime}$ appear in this order along $H$. If $a c^{\prime} \notin E(G)$, then $x a B b^{\prime}, x c B b^{\prime}$, and $x b C c^{\prime} y b^{\prime}$ induce a theta, so $a c^{\prime} \in E(G)$. Symmetrically, $c^{\prime} b, b a^{\prime}, a^{\prime} c, b^{\prime} c$, and $b^{\prime} a$ are all 
in $E(G)$, so $H, x$, and $y$ induce a cube, a contradiction to (1). It follows that every vertex has neighbors in at most two sectors of $W$.

If $G=W$, then $G$ is basic, so let $L$ be a component of $G \backslash W$. Note that $N(L)$ contains at least two vertices since $G$ has no clique separator. If $N(L)$ is included in a sector of $W$, then the ends of this sector form a proper separator. We may therefore assume that $N(L)$ intersects at least two sectors of $W$.

Since $L$ is connected, it contains a path $P=u \ldots v$ such that $u$ has neighbors in a sector of $W$ (say $C$ up to symmetry), and $v$ has neighbors in another sector of $W$ (say $A$ up to symmetry). Suppose that $P$ is minimal with respect to this property. Then either $u=v$ and by the second paragraph of this proof, $u$ has no neighbor in $B$; or $u \neq v$ and, by minimality of $P, u$ has neighbors only in $C, v$ has neighbors only in $A$, and the interior of $P$ is anticomplete to $W$. In each case, we let $u^{\prime}$ be the neighbor of $u$ in $C$ closest to $a$ along $C$ and we let $v^{\prime}$ be the neighbor of $v$ in $A$ closest to $c$ along $A$. Note that because $u^{\prime}$ and $v^{\prime}$ exist, $a b \notin E(G)$ and $b c \notin E(G)$. So, $a c \notin E(G)$ for otherwise, $(W, x)$ would form a pyramid and be a non-proper wheel. Now, the three paths $a x c, B$, and $a C u^{\prime} u P v v^{\prime} A c$ form a theta, a contradiction. This proves (2).

(3) We may assume that $G$ does not contain an extended prism.

Proof of (3). Let $W$ be an extended prism in $G$, with notation as in the definition. Suppose that some vertex $z$ of $G \backslash W$ has neighbors in three distinct paths among $A, A^{\prime}, B, B^{\prime}$, and $C$, and call $Q, R, S$ these three paths (so $\{Q, R, S\} \subseteq\left\{A, A^{\prime}, B, B^{\prime}, C\right\}$ ). It is easy to check that some hole $H$ of $W$ contains $Q$ and $R$. By Lemma 4.1, $z$ must have three neighbors in $H$, so $H$ and $z$ form a proper wheel, a contradiction to (2).

If $G=W$, then $G$ is basic, so let $L$ be a component of $G \backslash W$. Note that $N(L)$ contains at least two vertices since $G$ has no clique separator. If $N(L)$ is included in one of $V(A), V\left(A^{\prime}\right), V(B), V\left(B^{\prime}\right)$, or $V(C)$, then the ends of this path form a proper separator. We may therefore assume that $N(L)$ intersects at least two paths in $\left\{A, A^{\prime}, B, B^{\prime}, C\right\}$.

Since $L$ is connected, it contains a path $P=u \ldots v$ such that $u$ has neighbors in a path $Q \in\left\{A, A^{\prime}, B, B^{\prime}, C\right\}$ and $v$ has neighbors in another path $R \in\left\{A, A^{\prime}, B, B^{\prime}, C\right\}$. Suppose that $P$ is minimal with respect to this property. So by the minimality of $P$, either $u=v$ and by the second paragraph of this proof, $u=v$ has no neighbor in $\left\{A, A^{\prime}, B, B^{\prime}, C\right\} \backslash\{Q, R\}$; or $u \neq v$ and $u$ has neighbors only in $Q, v$ has neighbor only in $R$ and the interior of $P$ is anticomplete to $W$.

Note that each of $N_{Q}(u)$ and $N_{R}(v)$ is a vertex or an edge. For otherwise, suppose that $u$ has two non-adjacent neighbors in $Q$ (resp. in $R$ ). Since $G$ is subcubic and $Q$ (resp. $R$ ) can be completed to a hole $J$ of $W$, by Lemma 4.1 . $u$ has three pairwise non-adjacent neighbors in $J$, so $G$ contains a proper wheel, a contradiction to (2). We may now break into four cases. 
Case 1: $\{Q, R\}=\left\{A, A^{\prime}\right\}$ or $\{Q, R\}=\left\{B, B^{\prime}\right\}$. Up to symmetry, we suppose $Q=A$ and $R=A^{\prime}$. Then, $P$ can be used to find a path from $a$ to $a^{\prime}$ that does not contain $x$, and that together with $B, B^{\prime}$ and $C$ form a prism, a contradiction.

Case 2: $\{Q, R\}=\{A, B\}$ or $\{Q, R\}=\left\{A^{\prime}, B^{\prime}\right\}$. Up to symmetry, we suppose $Q=A$ and $R=B$. If $u$ has two adjacent neighbors in $A$, then $A$, $A^{\prime}, C$, a subpath of $B$, and $P$ form a prism. So, $u$ has exactly one neighbor in $A$, and symmetrically, $v$ has exactly one neighbor in $B$. So, $A, B$, and $P$ form a theta.

Case 3: $\{Q, R\}=\left\{A, B^{\prime}\right\}$ or $\{Q, R\}=\left\{B, A^{\prime}\right\}$. Up to symmetry, we suppose $Q=A$ and $R=B^{\prime}$. If $u$ has two adjacent neighbors in $A$, then $A$, $A^{\prime}, C$, a subpath of $B^{\prime}$, and $P$ form a prism. So, $u$ has exactly one neighbor in $A$, and symmetrically, $v$ has exactly one neighbor in $B^{\prime}$. So, $A, B^{\prime}, C$, and $P$ form a theta.

Case 4: $\{Q, R\}$ is one of $\{A, C\},\left\{A^{\prime}, C\right\},\{B, C\}$ or $\left\{B^{\prime}, C\right\}$. Up to symmetry, we suppose $Q=A$ and $R=C$. If $v$ has two adjacent neighbors in $C$, then $C, B, B^{\prime}$, a subpath of $A$ and $P$ form a prism. So, $v$ has exactly one neighbor in $C$. So, $C, B, A^{\prime}$, a subpath of $A$, and $P$ form a theta.

This proves (3).

(4) We may assume that $G$ does not contain a pyramid.

Proof of (4). Let $W$ be a pyramid with notation as in the definition. First note that a vertex $v \in V(G \backslash W)$ cannot have neighbors in the three paths $P_{1}, P_{2}$, and $P_{3}$, for otherwise there exists a theta from $v$ to $x$.

If $G=W$, then $G$ is basic, so let $L$ be a component of $G \backslash W$. Note that $N(L)$ contains at least two vertices since $G$ has no clique separator. If $N(L)$ is included in one of $P_{1}, P_{2}$, or $P_{3}$, then the ends of this path form a proper separator, or a clique separator when this path has length 1 . We may therefore assume that $N(L)$ intersects at least two paths.

So, since $L$ is connected, it contains a path $P=u \ldots v$ such that $u$ has neighbors in a path $P_{i}$ (say $P_{1}$ up to symmetry), and $v$ has neighbors in another path $P_{j}$ (say $P_{2}$ up to symmetry). Suppose that $P$ is minimal with respect to this property. So by minimality, either $u=v$ and by the first paragraph of this proof, $u=v$ has no neighbor in $P_{3}$; or $u \neq v$ and $u$ has neighbors only in $P_{1}, v$ has neighbor only in $P_{2}$, and the interior of $P$ is anticomplete to $W$.

Note that each of $N_{P_{1}}(u)$ and $N_{P_{2}}(v)$ is a vertex or an edge. If $u=v$, this is because $G$ contains no proper wheel by (2). If $u \neq v$, this is because $u$ and $v$ have degree at most 3 and we apply Lemma 4.1 .

If $N_{P_{1}}(u)$ and $N_{P_{2}}(v)$ are both edges, then $u \neq v$ (because $G$ is subcubic), so $P_{1}, P_{2}$, and $P$ form a prism. If each of $N_{P_{1}}(u)$ and $N_{P_{2}}(v)$ is a vertex, then $P_{1}, P_{2}$, and $P$ form a theta. So, up to symmetry, $N_{P_{1}}(u)$ is a vertex 
$u^{\prime}, N_{P_{2}}(v)$ is an edge $y z$ (where $x, y, z, a_{2}$ appear in this order along $P_{2}$ ). If $u^{\prime} \neq x_{1}$, then $V(P) \cup V(W) \backslash V\left(z P_{2} a_{2}\right)$ induces a theta from $u^{\prime}$ to $x$, so $u^{\prime}=x_{1}$. Hence, $W$ and $P$ form an extended prism, a contradiction to (3) This proves (4).

(5) We may assume that $G$ does not contain a hole.

Proof of (5). Let $W$ be a hole in $G$. First note that a vertex $v \in V(G \backslash W)$ cannot have three neighbors in $W$, for otherwise $v$ and $W$ would form a proper wheel or a pyramid. So, by Lemma 4.1, every vertex of $G \backslash W$ has at most one neighbor in $W$, or exactly two neighbors in $W$ that are adjacent.

If $G=W$, then $G$ is basic, so suppose that $L$ is a component of $G \backslash W$. If $N(L)$ is included in some edge of $W$, then $G$ has a clique separator, so suppose that there exist $a, b \in V(W)$ that are non-adjacent and that both have neighbors in $L$. Since $L$ is connected, there exists a path $P=u \ldots v$, such that $u$ is adjacent to $a$ and $v$ is adjacent to $b$. We suppose that $a, b, u, v$ and $P$ are chosen subject to the minimality of $P$. Note that $u \neq v$ since a vertex in $G \backslash W$ cannot have two non-adjacent neighbors in $W$.

Suppose that some internal vertex of $P$ has a neighbor $x$ in $W$. So $x$ must be adjacent to $a$, for otherwise a subpath of $P$ from $u$ to a neighbor of $x$ in $P$ contradicts the minimality of $P$. Similarly, $x$ is adjacent to $b$. If $a$ and $b$ have two common neighbors in $H$, say $x$ and $y$ (so $W=a x b y a$ ), and $x$ and $y$ both have neighbors in the interior of $P$, then the vertices $x$ and $y$ together with a subpath of $P$ contradict the minimality of $P$. Hence, $x$ is the unique vertex of $W$ with neighbors in the interior of $P$. If $u$ and $v$ have exactly two adjacent neighbors in $W$, then $W$ and $P$ form an extended prism, a contradiction to (3). If exactly one of $u$ or $v$ has exactly two neighbors in $W$, then $W$ and a subpath of $P$ form a pyramid, a contradiction to (4). So, $u$ and $v$ both have a unique neighbor in $W$. Now, $P$ and $H$ form a proper wheel, a contradiction to (2).

So, the interior of $P$ is anticomplete to $W$. Hence, $P$ and $W$ form a theta, a prism or a pyramid, in every case a contradiction to $G \in \mathcal{C}$, or to (4). This proves (5).

(6) We may assume that $G$ does not contain a triangle.

Proof of (6). Let $W=a b c$ be a triangle in $G$. If $G=W$, then $G$ is basic, so suppose that $L$ is a component of $G \backslash W$. If $|N(L)| \leq 2$, then $G$ has a clique separator of size at most 2 , so suppose that $N(L)=\{a, b, c\}$.

Let $P=u \ldots v$ be a path in $L$ such that $u$ is adjacent to $a, v$ is adjacent to $b$, and suppose $P$ is minimal. If $u \neq v$, then $P, a$, and $b$ form a hole, a contradiction to (5), so $u=v$. By (1), $u$ is non-adjacent to $c$. Hence, a path in $L$ from $u$ to a neighbor of $c$, together with $a$, would form a hole, a contradiction to (5). This proves (6).

Now, by (5) and (6), $G$ has no cycle. So, $G$ is a tree. It is therefore a complete graph on at most two vertices (that is basic) or it a has clique 

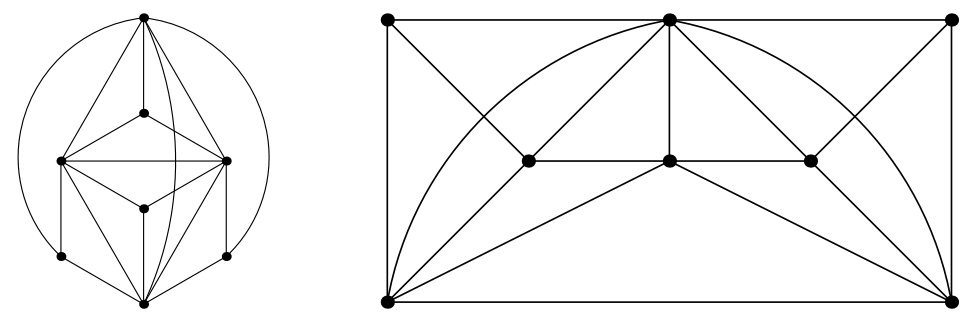

Figure 12: Two chordal graphs with clique number 4

separator of size 1.

Let us point out that Theorem 4.2 is a full structural description of the class of subcubic (theta, prism)-free graphs, in the sense that every graph in the class can be obtained from basic graphs by repeatedly applying some operations: gluing along a (possibly empty) clique, and an operation called proper gluing that we describe now.

Consider two graphs $G_{1}$ and $G_{2}$. Suppose that $G_{1}$ contains two nonadjacent vertices $a_{1}$ and $b_{1}$ of degree 3 , and such that a path $P_{1}$ from $a_{1}$ to $b_{1}$ with internal vertices all of degree 2 exists in $G_{1}$. Suppose that $G_{2}$ contains two non-adjacent vertices $a_{2}$ and $b_{2}$ of degree 2 , and such that a path $P_{2}$ from $a_{2}$ to $b_{2}$ with internal vertices all of degree 2 exists in $G_{2}$. Let $G$ be the graph obtained from the disjoint union of $G_{1}$ and $G_{2}$ by removing the internal vertices of $P_{1}$ and $P_{2}$, by identifying $a_{1}$ and $a_{2}$, and by identifying $b_{1}$ and $b_{2}$. We say that $G$ is obtained from $G_{1}$ and $G_{2}$ by a proper gluing.

We omit the details of the proof and just sketch it. We apply Theorem 4.2. If $G$ is basic, there is nothing to prove. If $G$ has a clique separator, it is obtained by two smaller graphs by gluing along a clique. If $G$ has a proper separation, then it is obtained from smaller graphs by a proper gluing.

Corollary 4.3. Every subcubic (theta, prism)-free graph (and therefore every even-hole-free subcubic graph) has tree-width at most 3.

PROOF - The proof is by induction. Let us first prove that all basic graphs have tree-width at most 3 . First observe that contracting an edge with one vertex of degree 2 preserves the tree-width. It follows that all basic graphs, except the cube and the extended prisms, have tree-width at most the tree-width of $K_{4}$, that is 3 . In Figure 12, we show a chordal graph $J$ with $\omega(J)=4$ that contains the cube or the smallest extended prism as a subgraph, showing that here again the tree-width is at most 3 .

Also, it is easy to check that the two operations gluing along a clique and proper gluing do not increase the tree-width (this can be proved by observing that the operation are particular cases of what is called clique- 
sum in the theory of tree-width, or by a direct proof using the definition of tree-width given at the beginning of the paper).

Note also that all graphs in $\mathcal{C}$ can be proved to be planar by an easy induction.

\section{5 (Even hole, pyramid)-free graphs with maximum degree at most 4}

Our goal in this section is to prove that (even hole, pyramid)-free graphs with maximum degree at most 4 have bounded tree-width. We rely on two known theorems that we now explain.

Let $H$ be a hole in a graph and let $u$ be a vertex not in $H$. We say that $u$ is major w.r.t. $H$ if $N_{H}(u)$ is not included in a 3-vertex path of $H$. We omit "w.r.t. $H$ " when $H$ is clear from the context.

Lemma 5.1. If $G$ be an (even hole, pyramid)-free graph with maximum degree at most $4, H$ is a hole of $G$ and $v$ is a vertex that is major w.r.t. $G$, then $v$ has exactly three neighbors that are pairwise non-adjacent.

PROOF - Since $v$ is major, it has at least two neighbors in $H$. If $v$ has exactly two neighbors in $H$, since $v$ is major these two neighbors are nonadjacent. Therefore, $H$ and $v$ form a theta, a contradiction. If $G$ has exactly three neighbors in $H$, then they are pairwise non-adjacent because $v$ is major and $G$ has no pyramid. If $v$ has 4 neighbors in $H$, then $H$ and $v$ form an even wheel, a contradiction.

When $H$ is a hole in some graph and $u$ is a vertex not in $H$ with at least two neighbors in $H$, we call $u$-sector of $H$ any path of $H$ of length at least 1 , whose ends are adjacent to $u$ and whose internal vertices are not. Observe that $H$ is edgewise partitioned into its $u$-sectors.

Note that by Lemma 5.1, when $v$ is major w.r.t. $H,(H, v)$ is a wheel, so the notion of $v$-sector in $H$ is equivalent to the notion of a sector the wheel $(H, v)$. The following appeared in [8].

Theorem 5.2. Let $G$ be a graph with no even hole and no pyramid, $H$ a hole in $G$ and $v$ a major vertex w.r.t. $H$. If $C$ is a connected component of $G \backslash N[v]$, then there exists a v-sector $P=x \ldots y$ of $H$ such that $N(C) \subseteq$ $\{x, y\} \cup(N(v) \backslash V(H))$.

A graph $G$ is a ring if its vertex-set can be partitioned into $k \geq 3$ sets $X_{1}, \ldots, X_{k}$ such that (the subscript are taken modulo $k$ ):

1. $X_{1}, \ldots, X_{k}$ are cliques;

2. for all $i \in\{1, \ldots, k\}, X_{i}$ is anticomplete to $V(G) \backslash\left(X_{i-1} \cup X_{i} \cup X_{i+1}\right)$; 
3. for all $i \in\{1, \ldots, k\}$, some vertex of $X_{i}$ is complete to $X_{i-1} \cup X_{i+1}$;

4. for all $i \in\{1, \ldots, k\}$ and all $x, x^{\prime} \in X_{i}$, either $N[x] \subseteq N\left[x^{\prime}\right]$ or $N\left[x^{\prime}\right] \subseteq$ $N[x]$.

A graph $G$ is a 7-hyperantihole if its vertex-set can be partitioned into 7 sets $X_{1}, \ldots, X_{7}$ such that (the subscript are taken modulo $k$ ):

1. $X_{1}, \ldots, X_{k}$ are cliques;

2. for all $i \in\{1, \ldots, k\}, X_{i}$ is complete to $V(G) \backslash\left(X_{i-1} \cup X_{i} \cup X_{i+1}\right)$;

3. for all $i \in\{1, \ldots, k\}, X_{i}$ is anticomplete to $X_{i-1} \cup X_{i+1}$.

The following is a rephrasing of Theorem 1.8 in [5]. Note that in [5], the definition of rings is slightly more restricted (at least 4 sets are required). We need rings with 3 sets for later use in inductions, and slightly extending the notion of ring cannot turn Theorem 1.8 in [5] into a false statement.

Theorem 5.3. If $G$ is (theta, prism, pyramid)-free and for every hole $H$ of $G$, no vertex of $G$ is major w.r.t. $H$, then $G$ is a complete graph, or $G$ is a ring, or $G$ is a "-hyperantihole, or $G$ has a clique separator.

Lemma 5.4. A complete graph, a ring, or a 7-antihole of maximum degree at most 4 does not contain $K_{6}$ as a minor.

PROOF - For complete graphs, this is obvious since $K_{5}$ is the biggest complete graph of maximum degree at most 4 . For 7 -hyperantiholes, the proof is also easy because each of the cliques in the definition must be on a single vertex, so that $|V(G)|=7$, and a $K_{6}$ minor obviously does not exists.

So, suppose that $G$ is a ring of maximum degree at most 4 (we use for $G$ the notation as in the definition of rings).

(1) For all $i \in\{1, \ldots, k\}$, one of $X_{i-1}, X_{i}$ or $X_{i+1}$ contains only one vertex. Moreover, if $\left|X_{i}\right|=3$, then $X_{i-1}, X_{i+1}$ and $X_{i+2}$ all contain only one vertex.

Proof of (1). Otherwise, some vertex in $X_{i}$ or $X_{i+1}$ has degree 5, a contradiction to our assumption. This proves (1).

We now prove by induction on $k$ (the number of sets in the ring) that $G$ does not contain $K_{6}$ as a minor. If $k$ equals 3 or 4 , then by (1), we see that $|V(G)| \leq 6$, so $G$ does not contain $K_{6}$ as a minor since $G$ is not $K_{6}$. If $k \geq 5$, then by (1), there exist two distinct sets of the ring $X_{i}, X_{j}$ that are anticomplete to each other and such that $X_{i}=\{x\}$ and $\left|X_{j}\right|=\left\{x^{\prime}\right\}$. By the definition of rings, $G \backslash\left\{x, x^{\prime}\right\}$ has two connected components $C$ and $C^{\prime}$, and it is straightforward to check the two graphs $G_{C}$ and $G_{C^{\prime}}$ obtained from $G\left[C \cup\left\{x, x^{\prime}\right\}\right]$ and $G\left[C^{\prime} \cup\left\{x, x^{\prime}\right\}\right]$ respectively by adding an edge between $x$ and $x^{\prime}$ are rings (this is the place where we need a ring on three sets). Also, 
it is straightforward to check that a $K_{6}$ minor in $G$ yields a $K_{6}$ minor in one of $G_{C}$ or $G_{C^{\prime}}$, a contradiction to the induction hypothesis.

We can now prove the main theorem of this section.

Theorem 5.5. If a graph $G$ is (even hole, pyramid)-free with maximum degree 4 , then $G$ contains no $K_{6}$ as a minor.

PROOF - Suppose that $G$ is a counter-example with a minimum number of vertices. So, $G$ contains $K_{6}$ as a minor. By the minimality of $G$ and the definition of minors, it follows that $V(G)$ can be partitioned into six nonempty sets $B_{1}, \ldots, B_{6}$ such that for all $i, j \in\{1, \ldots, 6\}, G\left[B_{i}\right]$ is connected and there is at least one edge between $B_{i}$ and $B_{j}$.

Case 1: $G$ contains no hole with a major vertex.

By Theorem 5.3 and Lemma 5.4, $G$ has a clique separator $K$. It is straightforward to check that for one component $C$ of $G \backslash K$, the graph $G[K \cup C]$ contains $K_{6}$ as a minor, a contradiction to the minimality of $G$.

Case 2: $G$ contains a hole $H$ and a vertex $v$ that is major w.r.t. $H$.

By Lemma 5.1, $v$ has exactly three neighbors in $H$ that are pairwise non-adjacent. Possibly, $v$ has a neighbor $w \notin H$ (if $v$ has degree three, we set $v=w)$. Let $a, b, c$ be the three neighbors of $v$ in $H$.

Up to symmetry, $B_{5}$ and $B_{6}$ do not contain $a, b, c, w$. So, some connected component $C$ of $G \backslash\{v, w, a, b, c\}$ contains $B_{5} \cup B_{6}$. By Theorem 5.2, there exists a $v$-sector, say $P=a \ldots b$ up to symmetry, of $H$ such that $N(C) \subseteq$ $\{a, b, w\}$. Note that if $v=w$, then $\{a, b\}$ is a separator of $G$, in which case the proof is easier. So in what follow, a reader may assume for simplicity that $v \neq w$, though what is written is correct even if $v=w$.

Let $C^{\prime}$ be the union of all components $X$ of $G \backslash\{v, w, a, b, c\}$ such that $N(X) \subseteq\{a, b, w\}$. Let $D$ be $V(G) \backslash\left(C^{\prime} \cup\{a, b, w\}\right)$. Note that $C \subseteq C^{\prime}$ and $v, c \in D$. Note that $B_{5} \cup B_{6} \subseteq C^{\prime}$, and since $\{a, b, w\}$ separates $C^{\prime}$ from $D$, we may assume that $B_{4} \subseteq C^{\prime}$.

Let $S_{a}$ (resp. $S_{b}$ ) be the $v$-sector of $H$ from $a$ (resp. $b$ ) to $c$. Let $G^{\prime}$ be the graph obtained from $G\left[C^{\prime} \cup\{a, b, c, v, w\}\right]$ by adding the edges $c a$ and $c b$. Also, the edge $c w$ is added to $G^{\prime}$ if and only if $w$ has a neighbor in the interior of the path formed by $S_{a}$ and $S_{b}$.

(1) $G^{\prime}$ is (even hole, pyramid)-free and has maximum degree at most 4.

Proof of (1). Clearly $G$ has maximum degree at most 4 . Since $G^{\prime} \backslash c$ is an induced subgraph of $G$, every even hole or pyramid of $G^{\prime}$ goes through $c$.

Suppose that $J$ is an even hole of $G^{\prime}$. Since it goes through $c$, up to symmetry, we may assume that $J$ goes through $c b$. If $J$ contains $a$, then $G$ is formed of $a, c, b$ and a path $P$ of even length from $a$ to $b$. So, $P, S_{a}$ and $S_{b}$ form an even hole of $G$, unless $w \in V(J)$ and $w$ has a neighbor in the interior of the path induced by $S_{a} \cup S_{b}$. But this case leads to a contradiction, since 
by the definition of $G^{\prime}$, we would have $c w \in E\left(G^{\prime}\right)$, so $J$ would not be a hole of $G^{\prime}$.

So, $J$ does not go through $a$. It follows that $J$ is formed by $b c$ and a path $Q$ of odd length from $w$ to $b$. Note that in this case, $c w \in E(G)$ and $v \notin V(J)$, so $v \neq w$. It follows that $Q$ and $v$ form an even hole of $G^{\prime}$, a contradiction.

Suppose that $G^{\prime}$ contains a pyramid $\Pi$. Since $\Pi$ contains $c$, it does not contain $v$ because $v$ dominates $c$, in $G^{\prime}$, ie $N_{G^{\prime}}[c] \subseteq N_{G^{\prime}}[v]$ (and in a pyramid, no vertex dominates another vertex). If we replace $c$ by $v$ in $\Pi$, then we obtain an induced subgraph of $G$ that is not a pyramid since $G$ is pyramid-free. This implies that $c w \notin E\left(G^{\prime}\right)$. So, $c$ has degree 2 in $\Pi$ and $w$ has no neighbor in the interior of the path induced by $S_{a} \cup S_{b}$. Hence, replacing $a c b$ by $S_{a}$ and $S_{b}$ in $\Pi$ yields a pyramid of $G$, a contradiction. This proves (1).

(2) $G^{\prime}$ contains $K_{6}$ as a minor.

Proof of (2). Suppose that $a \in B_{1}, w \in B_{2}$ and $b \in B_{3}$. We then set $B_{1}^{\prime}=\left(B_{1} \backslash D\right) \cup\{c\}, B_{2}^{\prime}=\left(B_{2} \backslash D\right) \cup\{v\}$ and $B_{3}^{\prime}=B_{3} \backslash D$. We observe that there are edges from $B_{1}^{\prime}$ to $B_{2}^{\prime}$, from $B_{1}^{\prime}$ to $B_{3}^{\prime}$ and from $B_{2}^{\prime}$ to $B_{3}^{\prime}$. Also, each of these sets is connected in $G^{\prime}$, and together with $B_{4}, B_{5}$ and $B_{6}$ they form a $K_{6}$ minor of $G^{\prime}$. We may therefore assume that $B_{3} \cap\{a, b, w\}=\emptyset$, so that $B_{3} \subseteq C^{\prime}$.

If $\{a, b, w\} \cap B_{1}=\{a, b\}$, then $\{a, b, w\} \cap B_{2}=\{w\}$. We then set $B_{1}^{\prime}=\left(B_{1} \backslash D\right) \cup\{c\}$ and $B_{2}^{\prime}=\left(B_{2} \backslash D\right) \cup\{v\}$. We observe that there are edges from $B_{1}^{\prime}$ to $B_{2}^{\prime}$. Also, these sets are connected in $G^{\prime}$, and together with $B_{3}, B_{4}, B_{5}$ and $B_{6}$ they form a $K_{6}$ minor of $G^{\prime}$. Hence, we may assume that $\{a, b, w\} \cap B_{1} \neq\{a, b\}$, and symmetrically $\{a, b, w\} \cap B_{2} \neq\{a, b\}$.

We may assume that $a, w \in B_{1}$. We set $B_{1}^{\prime}=\left(B_{1} \backslash D\right) \cup\{c, v\}$ and $B_{2}^{\prime}=B_{2} \backslash D$. We observe that there are edges from $B_{1}^{\prime}$ to $B_{2}^{\prime}$. Also, these sets are connected in $G^{\prime}$, and together with $B_{3}, B_{4}, B_{5}$ and $B_{6}$ they form a $K_{6}$ minor of $G^{\prime}$. This proves (2).

Since $G^{\prime}$ is smaller than $G,(1)$ and (2) contradict the minimality of $G$.

In the next corollary, we use the function $f_{H}(k)$ as defined in Theorem 1.1 .

Corollary 5.6. Every (even hole, pyramid)-free graph of maximum degree at most 4 has tree-width less than $f_{K_{6}}(3)$.

PROOF - Suppose that $G$ has tree-width at least $f_{K_{6}}(3)$. By Theorem 5.5 . $G$ does not contain $K_{6}$ as a minor. By Theorem 1.1. $G$ contains a $(3 \times 3)$ wall or the line graph of a chordless $(3 \times 3)$-wall as an induced subgraph. This yields a contradiction because the $(3 \times 3)$-wall contains a theta, and the line graph of a chordless $(3 \times 3)$-wall contains a prism (see Figure 13 , a contradiction to Lemma 2.1 . 

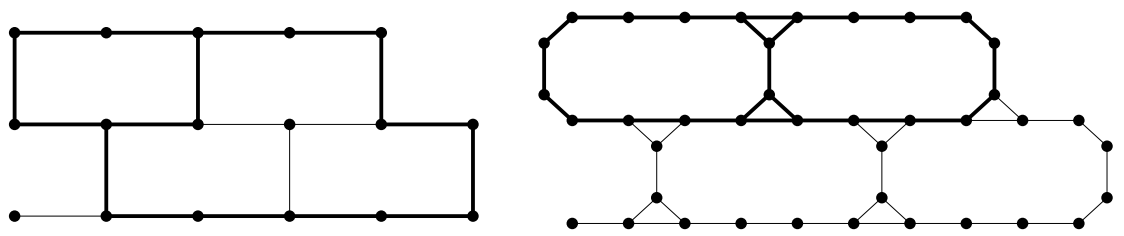

Figure 13: A theta in $(3 \times 3)$-wall and a prism in the line graph of a chordless $(3 \times 3)$-wall

This approach might work for maximum degree 5 with much more technicalities, but for larger values it fails as far as we can see.

\section{A possible structure theorem for even-hole-free graphs with maximum degree at most 4}

In this section, we investigate a possible structure theorem that would describe even-hole-free graphs with maximum degree at most 4 . We call patterns, the graphs that are represented on Figure 14 and Figure 15. Say that a graph is basic if it is a complete graph or a chordless cycle, or it can be obtained from one of the patterns, by replacing dashed lines with paths of length at least one or contracting some dashed lines into single vertices. We believe that an even-hole-free graph with maximum degree 4 must be either basic or decomposable with a clique separator or a 2-join that we define below.

A 2-join in a graph $G$ is a partition of $V(G)$ into two sets $X_{1}, X_{2}$ each of size at least 3 , such that for $i=1,2, X_{i}$ contains two non-empty disjoint sets $A_{i}, B_{i}, A_{1}$ is complete to $A_{2}, B_{1}$ is complete to $B_{2}$, and there are no other edges between $X_{1}$ and $X_{2}$. Moreover, for $i=1,2, X_{i}$ does not consist of a path with one end in $A_{i}$, one end in $B_{i}$ and no internal vertex in $A_{i} \cup B_{i}$.

We are not sure that our list of patterns is complete for our class, but we believe that the real list is close to it and, above all, finite. This should imply that the tree-width is bounded. Also, we wonder whether a similar approach can be extended to even-hole-free graphs of maximum degree $k$ for any fixed integer $k$. Observe that for $k=3$, this is what we actually do in Theorem 4.2, since the list of basic graphs can be seen as obtained by a finite list of patterns and the so-called proper separator is a special case of 2 -join. For $k \geq 5$, rings (already defined in Section 5) become a problem, but an extension of the notion of 2 -join might lead to a true statement.

\section{References}

[1] Isolde Adler and Frederik Harwath. Property testing for bounded degree databases. In Rolf Niedermeier and Brigitte Vallée, editors, 35th 


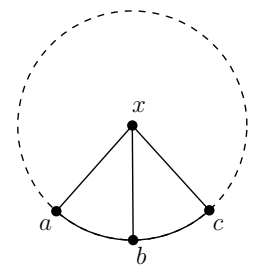

(a)

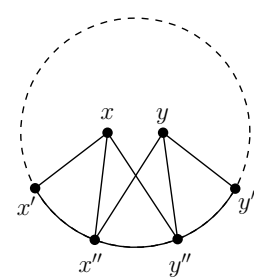

(e)

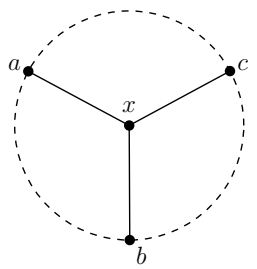

(b)

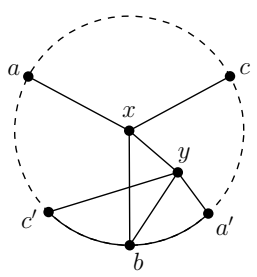

(f)

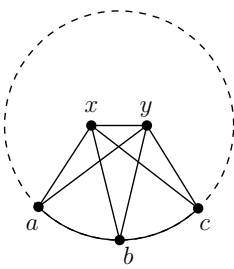

(c)

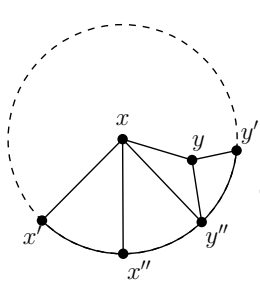

(g)

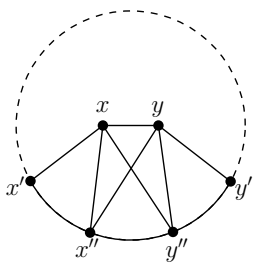

(d)

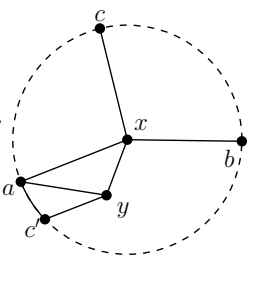

(h)

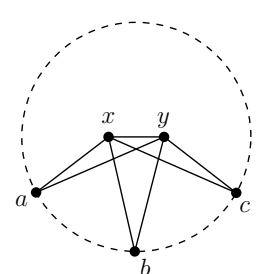

(i)

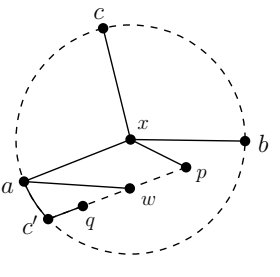

(j)

Figure 14: Patterns in the pyramid-free case (solid lines represent edges)

Symposium on Theoretical Aspects of Computer Science, STACS 2018, February 28 to March 3, 2018, Caen, France, volume 96 of LIPIcs, pages 6:1-6:14. Schloss Dagstuhl - Leibniz-Zentrum fuer Informatik, 2018 .

[2] Lowell Beineke and Allen Schwenk. On a bipartite form of the ramsey problem. Proc. 5th British Combinatorial Conference 1975, Congressus Numer. XV, pages 17-22, 1975.

[3] Itai Benjamini, Oded Schramm, and Asaf Shapira. Every minorclosed property of sparse graphs is testable. Advances in mathematics, 223(6):2200-2218, 2010.

[4] Hans L. Bodlaender and Dimitrios M. Thilikos. Treewidth for graphs with small chordality. Discrete Applied Mathematics, 79(1-3):45-61, 1997.

[5] Valerio Boncompagni, Irena Penev, and Kristina Vuskovic. Cliquecutsets beyond chordal graphs. Journal of Graph Theory, 91(2):192246, 2019. 


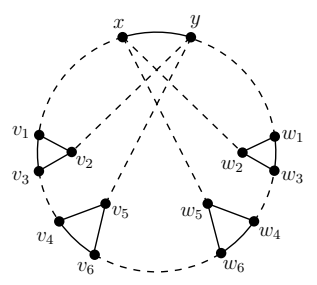

(a)

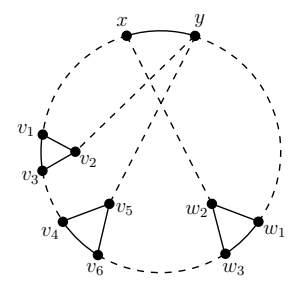

(d)

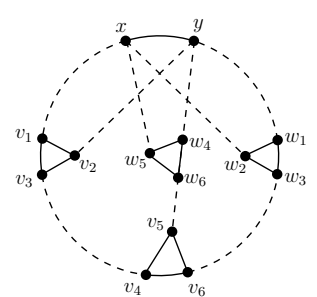

(b)

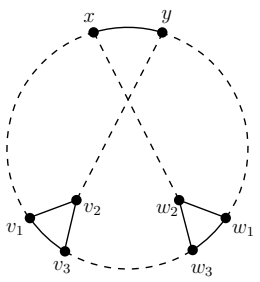

(e)

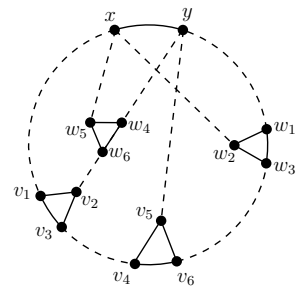

(c)

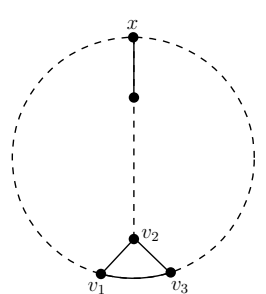

(f)

Figure 15: Patterns that contain pyramid

[6] Kathie Cameron, Steven Chaplick, and Chính T. Hoàng. On the structure of (pan, even hole)-free graphs. Journal of Graph Theory, 87(1):108-129, 2018.

[7] Kathie Cameron, Murilo V. G. da Silva, Shenwei Huang, and Kristina Vuskovic. Structure and algorithms for (cap, even hole)-free graphs. Discret. Math., 341(2):463-473, 2018.

[8] Maria Chudnovsky, Stéphan Thomassé, Nicolas Trotignon, and Kristina Vušković. Maximum independent sets in (pyramid, even hole)free graphs. CoRR, abs/1912.11246, 2019.

[9] Bruno Courcelle. The monadic second-order logic of graphs. i. recognizable sets of finite graphs. Inf. Comput., 85(1):12-75, 1990.

[10] Bruno Courcelle and Joost Engelfriet. Graph Structure and Monadic Second-Order Logic - A Language-Theoretic Approach, volume 138 of Encyclopedia of mathematics and its applications. Cambridge University Press, 2012.

[11] Fedor V. Fomin, Petr A. Golovach, and Dimitrios M. Thilikos. Contraction obstructions for treewidth. J. Comb. Theory, Ser. B, 101(5):302$314,2011$.

[12] Sebastian Forster, Danupon Nanongkai, Thatchaphol Saranurak, Liu Yang, and Sorrachai Yingchareonthawornchai. Computing and testing small connectivity in near-linear time and queries via fast local cut algorithms. SODA, 2020. 
[13] Oded Goldreich. Introduction to property testing. Cambridge University Press, 2017.

[14] Oded Goldreich and Dana Ron. Property testing in bounded degree graphs. Algorithmica, 32(2):302-343, 2002.

[15] Avinatan Hassidim, Jonathan A Kelner, Huy N Nguyen, and Krzysztof Onak. Local graph partitions for approximation and testing. In 2009 50th Annual IEEE Symposium on Foundations of Computer Science, pages 22-31. IEEE, 2009.

[16] Noleen Köhler Isolde Adler. On graphs of bounded degree that are far from being Hamiltonian. Submitted.

[17] Ken-ichi Kawarabayashi and Yuichi Yoshida. Testing subdivisionfreeness: property testing meets structural graph theory. In Proceedings of the forty-fifth annual ACM symposium on Theory of computing, pages 437-446. ACM, 2013.

[18] Akash Kumar, C Seshadhri, and Andrew Stolman. Random walks and forbidden minors ii: a poly $(\mathrm{d} \varepsilon-1)$-query tester for minor-closed properties of bounded degree graphs. In Proceedings of the 51st Annual ACM SIGACT Symposium on Theory of Computing, pages 559-567, 2019.

[19] Ilan Newman and Christian Sohler. Every property of hyperfinite graphs is testable. SIAM Journal on Computing, 42(3):1095-1112, 2013.

[20] Neil Robertson and Paul D. Seymour. Graph minors. v. excluding a planar graph. J. Comb. Theory, Ser. B, 41(1):92-114, 1986.

[21] Ana Silva, Aline Alves da Silva, and Cláudia Linhares Sales. A bound on the treewidth of planar even-hole-free graphs. Discrete Applied Mathematics, 158(12):1229-1239, 2010.

[22] Ni Luh Dewi Sintiari and Nicolas Trotignon. (theta, triangle)-free and (even hole, $\mathrm{k}_{4}$ )-free graphs. part 1 : Layered wheels. CoRR, abs/1906.10998, 2019.

[23] Andrew Thomason. On finite ramsey numbers. Eur. J. Comb., $3(3): 263-273,1982$.

[24] Kristina Vuskovic. Even-hole-free graphs: A survey. Applicable Analysis and Discrete Mathematics, 4, 102010.

[25] Yuichi Yoshida and Hiro Ito. Property testing on $k$-vertex-connectivity of graphs. Algorithmica, 62(3-4):701-712, 2012. 